\title{
Enhancing glycan presentation increases vaccine efficacy
}

Carrier proteins coupled to polysaccharides that are extracted from bacterial cell walls are used as vaccines to prevent many infectious diseases such as pneumonia and meningitis. These glycoconjugate vaccines elicit a $\mathrm{T}$ cell response that boosts the production of high-affinity antibodies against the polysaccharide by B cells. In a study published in Nature Medicine, Kasper and colleagues highlight the importance of the carbohydrate component in these vaccines. By maximizing the presentation of carbohydrate epitopes to T cells, they were able to construct a vaccine against group B streptococcus that is significantly more potent in a neonatal mouse model than

currently available vaccines. When bacterial capside polysaccharides are used as vaccines they fail to induce sustained T cell memory, which led researchers to the idea that $\mathrm{T}$ cell help is conferred by recognition of the protein portion of glycoconjugate vaccines. Indeed, proteins have typically been viewed as the only antigens presented by major histocompatibility complex II (MHCII) molecules to $\mathrm{T}$ cells. However, when the authors examined how glycoconjugates containing the type III polysaccharide of group B streptococcus (GBSIII) are processed and presented in antigen-presenting cells (APCs), they found that MHCII-associated carbohydrate epitopes are presented on the APC surface.

GBSIII is depolymerised into smaller glycans in endolysosomes by reactive oxygen species and it undergoes similar processing when coupled to a carrier protein such as ovalbumin (OVA). Interestingly, following such processing, MHCIIassociated carbohydrate was present on the surface of cells incubated with GBSIII-OVA but not on cells incubated with GBSIII alone, suggesting that peptides derived from the proteolytic digestion of the carrier protein are required to bind the glycan to the MHCII molecule and enable glycan presentation at the cell surface. The authors were also able to isolate CD4-expressing $\mathrm{T}$ cell clones that specifically recognized the carbohydrate portion of the glycoconjugate in the context of MHCII, and secreted interleukin-2 (IL-2) and IL-4, which aid antibody production by B cells. Using blocking antibodies against particular $\mathrm{T}$ cell receptor subtypes, they showed that this response was mediated by $\alpha \beta-T$ cell receptors.

To maximize the presentation of carbohydrate epitopes to T cells, and thus enhance the stimulation of the adaptive immune response, the authors designed a vaccine in which peptides were inserted at every eight repeating units of the polysaccharide. Compared with a standard GBSIIIOVA vaccine, this vaccine led to a marked increase in GBSIII-specific immunoglobulin $\mathrm{G}$ titres in mice. Importantly, all the pups of mice immunized with the modified glycoconjugate were protected when challenged with group B streptococcus immediately after birth, highlighting the vaccine's efficacy.

Whether the same adaptive response can be elicited in humans using glycoconjugates designed to maximize the generation of glycan epitopes remains to be investigated. Future crystallography studies should also shed light on the structural features of the ideal epitopes for these purposes and provide further details on the interactions between MHCII-bound glycans and T cell receptors. However, this study represents an important first step towards understanding glycoconjugate-induced immune responses, and improving the efficacy and immunogenicity of glycoconjugate vaccines.

Monica Hoyos Flight

ORIGINAL RESEARCH PAPER Avci, F. Y. et al.

A mechanism for glycoconjugate vaccine activation of the adaptive immune system and its implications for vaccine design. Nature Med. 17, 1602-1609 (2011) 